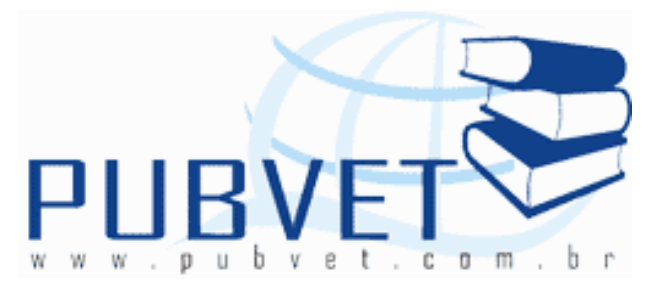

PUBVET, Publicações em Medicina Veterinária e Zootecnia.

\title{
Distribuição intraparenquimal da artéria hepática em tatu-peba (Euphractus sexcinctus) e tatu-verdadeiro (Dasypus novencinctus)
}

Andrezza Braga Soares da Silva ${ }^{1}$, Maria Michele Araújo de Sousa Cavalcante ${ }^{1}$, Sérgio Paulo Lima Guerra ${ }^{2}$, Fernando Augusto Tambelini Tizianel${ }^{3}$, Waldilleny Ribeiro de Araújo Moura², Marcia dos Santos Rizzo², Airton Mendes Conde Júnior ${ }^{4}$

${ }^{1}$ Acadêmica de Medicina Veterinária

${ }^{2}$ Médico(a) Veterinário(a)

${ }^{3}$ Biólogo, Diretor do Instituto Chico Mendes de Biodiversidade

${ }^{4}$ Prof. Dr. Adjunto do Departamento de Morfologia - UFPI

Médico Veterinário

\section{Resumo}

O tatu é um mamífero xenárthrico, juntamente com tamanduás e preguiças arborícolas. Aspectos morfológicos dos órgãos desses animais são carentes de informações que sirvam de base para a clínica de animais silvestres. Para isto, o presente trabalho visa descrever a distribuição arterial do parênquima hepático de tatus-peba e tatus-verdadeiro, levando em consideração sua lobação hepática. Foram utilizados 10 animais vítimas da caça predatória e doados à UFPI com autorização do SISBIO. Os mesmos foram dissecados e procedeu-se com a injeção de Látex Neoprene corado em vermelho na aorta 
SILVA, A.B.S. et al. Distribuição intraparenquimal da artéria hepática em tatu-peba (Euphractus sexcinctus) e tatu-verdadeiro (Dasypus novencinctus). PUBVET, Londrina, V. 8, N. 21, Ed. 270, Art. 1805, Novembro, 2014.

abdominal para visualização da ramificação arterial hepática. Por fim, os tatus foram fixados em solução de formaldeído tamponado a $10 \%$ e submetidos à dissecação para análise dos resultados. O fígado de ambas as espécies apresenta quatro lobos: direito, esquerdo, quadrado e caudado com seus processos papilar e caudado, e presença de vesícula biliar. Partindo da artéria hepática nos tatus analisados, esta emite ramos que irão vascularizar o fígado, porções inicial e final do duodeno e pâncreas. Ao ingressar no hilo hepático, esta artéria apresenta-se distribuída em dois ramos principais: direito e esquerdo, onde cada um vai inferir a presença de segmentação arterial própria nos lobos hepáticos, caracterizando, portanto, a segmentação anátomocirúrgica arterial. Além disso, há estreita relação entre metabolismo e dieta alimentar com a morfologia hepática e a distribuição vascular para este órgão.

Palavras-chave: tatu-peba, tatu-verdadeiro, fígado, artéria hepática

\title{
Intraparenchymal distribution of the hepatic artery in Sex-banded armadillo (Euphractus sexcinctus) and Nine-banded armadillo (Dasypus novencinctus)
}

\begin{abstract}
The armadillo is a xenárthrico mammal along with anteaters and arboreal sloths. Morphological aspects of the organs of these animals are lacking of information as a basis for clinical wild animals. Thus, this research aims to describe the arterial distribution of the hepatic parenchyma of sex-banded armadillo and nine-banded armadillo, assuming your liver lobation. Altogether were used 10 animals victims of poaching and donated to UFPI with permission of the SISBio. They were dissected and we proceeded with the injection of Neoprene latex red dyed in the abdominal aorta to visualize the hepatic arterial branch. Finally, the armadillos were fixed in formaldehyde solution buffered 10 $\%$ and underwent dissection for analysis of results. The liver of both species has four lobes: right, left, with their square and caudate caudate and papillary processes, and the presence of gallbladder. From hepatic artery in armadillos
\end{abstract}


SILVA, A.B.S. et al. Distribuição intraparenquimal da artéria hepática em tatu-peba (Euphractus sexcinctus) e tatu-verdadeiro (Dasypus novencinctus). PUBVET, Londrina, V. 8, N. 21, Ed. 270, Art. 1805, Novembro, 2014.

analyzed, this sends branches that will ventricularizing the liver, initial and final portions of the duodenum and pancreas. When entering the hepatic hilum, the artery presents distributed into two main branches, right and left, where each will infer the presence of segmentation own blood in the hepatic lobes, thus characterizing the anatomical and surgical arterial segmentation. Furthermore, there is a close relationship between diet and metabolism in liver morphology and vascular distribution for this organ.

Keywords: sex-banded armadillo, nine-banded armadillo, liver, hepatic artery.

\section{INTRODUÇÃO}

A morfologia dos animais selvagens é carente em literatura básica, principalmente quando se refere à espécies que apresentam algum potencial de exploração intensiva, seja como fonte alimentar, ou modelo biológico, como é o caso do tatu, mamífero Xenárthrico, ou seja, pertencente à superordem Xenarthra e o qual compõem a nossa fauna (MENEZES et al., 2001; ZIMBRES, 2010).

De acordo com Vizcaíno e Loughry (2008), os pertencentes da superordem Xenarthra compreendem os tatus (ordem Cigunlata), os tamanduás e as preguiças arborícolas (ordem Pilosa). Os membros da ordem Cigunlata caracterizam-se pela presença de osteodermos na pele, estruturas de aspecto ósseo que formam uma carapaça na pele de tatus. Esta estrutura óssea de origem dérmica está dividida em regiões de acordo com a sua localização e tem a função de ajudar contra a ação de predadores destes animais (PEREIRA JUNIOR, 2007).

Os tatus são importantes elos intermediários nas cadeias ou redes alimentares, pois se alimentam de pequenos seres como: insetos, minhocas e pequenos vertebrados, assim como frutas e raízes (PEREIRA JUNIOR, 2007; FISCHER et al., 2011). Apesar de ser tão importante para o ecossistema, o tatu vem sendo intensamente explorado pela humanidade como fonte de 
SILVA, A.B.S. et al. Distribuição intraparenquimal da artéria hepática em tatu-peba (Euphractus sexcinctus) e tatu-verdadeiro (Dasypus novencinctus). PUBVET, Londrina, V. 8, N. 21, Ed. 270, Art. 1805, Novembro, 2014.

alimento, remédios tradicionais, renda e ainda utilizados para atividades cinegéticas e usos em crenças populares (BARBOZA, 2009).

Esta situação têm despertado o interesse de pesquisadores em promover estudos laboratoriais em animais que venham à óbito pela caça predatória, com o intuito de enriquecer a bibliografia silvestre com informações relevantes para a clinica e manejo destes animais e ao mesmo tempo reduzir a sua exploração favorecendo que estas espécies sejam preservadas. Estes estudos são tão importantes que já se descobriu a capacidade de tatus em transmitir determinadas doenças ao ser humano, como a hanseníase, demonstrando a importância também para a saúde pública (DEPS et al., 2008).

Há poucos dados na literatura sobre as características morfofisiológicas dos diversos órgãos dos tatus, incluindo a descrição do aporte vascular para os diversos sistemas (AZEVEDO et al, 2008). O fígado, é o segundo maior órgão do corpo e a maior glândula (JUNQUEIRA \& CARNEIRO, 2008). Do ponto de vista funcional, é o local do organismo onde ocorrem numerosos processos metabólicos que dependem da existência de um aporte vascular hepático desenvolvido (BORGES et al., 2002; BARBINO et al., 2011).

Estudos recentes têm mostrado que os animais da ordem Xenarthra apresentam uma taxa metabólica reduzida, uma justificativa estaria na dieta alimentar desses animais, pobre em proteína (ZIMBRES, 2010). Assim, os órgãos responsáveis pelo metabolismo, como o fígado, devem ser cientificamente explorados para melhor compreensão das características de cada espécie.

Com isso, o presente trabalho visa estudar a morfologia macroscópica do fígado de tatus das espécies E.sexcinctus e D.novencinctus, e descrever a distribuição arterial para o mesmo. Obtidos os resultados, realizar possíveis comparações com animais da mesma superordem, como a preguiça, e explorar o mesmo trabalho em animais de outras ordens. Com isso, apresentar bases anatômicas que favoreçam os avanços na clínica e manejo desses animais, especialmente os que chegam às unidades de conservação sem atendimento prévio. 
SILVA, A.B.S. et al. Distribuição intraparenquimal da artéria hepática em tatu-peba (Euphractus sexcinctus) e tatu-verdadeiro (Dasypus novencinctus). PUBVET, Londrina, V. 8, N. 21, Ed. 270, Art. 1805, Novembro, 2014.

\section{MATERIAL E MÉTODOS}

Foram selecionados 10 (dez) tatus adultos, machos e fêmeas, que vieram à obito vítimas da caça predatória no município de Serra da Capivara apreendidos pelo Instituto Chico Mendes de Conservação da Biodiversidade (ICMBio-PI). Os animais foram doados e previamente encaminhados ao laboratório de Embriologia e Histotécnica do Departamento de Morfologia da Universidade Federal do Piaui (UFPI).

Partindo de suas informações preliminares, como a espécie a qual pertencem, foi iniciada a dissecação realizando uma incisão na região ventral do tórax, onde pele e músculos ventro-laterais foram rebatidos, seguido da remoção parcial do esterno e de algumas costelas do antímero esquerdo, para permitir a visualização e canulação da artéria aorta torácica no sentido caudal. $\mathrm{Na}$ sequencia, iniciou-se a injeção do sistema vascular com Neoprene látex corado com pigmento vermelho (Hidracor $®$ ) para melhor identificação dos vasos arteriais perante as estruturas adjacentes.

Logo após a injeção, os espécimes foram fixados, por meio de infusão intramuscular, subcutânea e intracavitária, de solução aquosa de formaldeído a $10 \%$, com posterior submersão dos animais na mesma solução fixadora, por um período mínimo de sete dias. Em seguida, as artérias abdominais foram dissecadas a partir de incisão na cavidade abdominal ao nível da linha média, desde a cartilagem xifóide até próximo a genitália externa. Características topográficas dos órgãos foram observadas para posterior afastamento lateral das vísceras iniciando a evidencialização da aorta abdominal e de seus ramos colaterais.

O fígado de cada espécie estudada foi destacado de sua localização na cavidade abdominal e conservado em solução de formol a $10 \%$ para visualização de aspectos morfológicos e descrição dos ramos da artéria hepática que adentram o parenquima do órgão. Foram registras fotografias dos espécimes, da cavidade abdominal e do fígado dos tatus utilizando Máquina Digital Sony modelo DSC - H10. Toda a nomenclatura adotada foi 
SILVA, A.B.S. et al. Distribuição intraparenquimal da artéria hepática em tatu-peba (Euphractus sexcinctus) e tatu-verdadeiro (Dasypus novencinctus). PUBVET, Londrina, V. 8, N. 21, Ed. 270, Art. 1805, Novembro, 2014.

baseada no proposto pelo International Committee on Veterinary Gross Anatomical Nomenclature (2012).

\section{RESULTADOS E DISCUSSÃO}

O fígado dos tatu-peba e tatu-verdadeiro apresenta-se dividido em quatro lobos: direito, esquerdo, quadrado e caudado com seus processos papilar e caudado, e presença de vesícula biliar em contato com a face visceral do órgão (Figura.1.B). Na face diafragmática, observa-se a veia cava caudal e as veias hepáticas, que irão desembocar neste vaso, além do ligamento redondo que percorre o lobo quadrado nesta mesma face (Figura.1.A). Já na face visceral, encontra-se a vesícula biliar (em verde) e a artéria hepática, com seus ramos direito e esquerdo penetrando no parênquima (Figura.1.B).

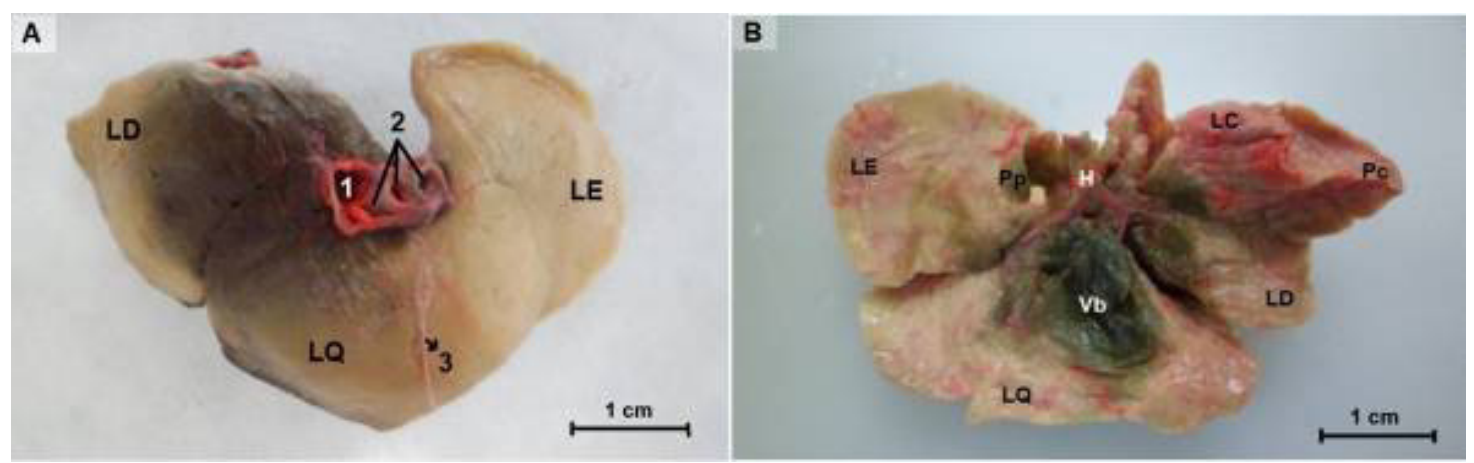

Figura.1. Prancha fotográfica do fígado do tatu (Euphractus sexcintus) mostrando suas faces diafragmática (A) e visceral (B). 1- Veia cava caudal. 2Veias hepáticas. 3- Ligamento redondo . LD- Lobo direito. LE- Lobo esquerdo. LQ- Lobo quadrado. LC- Lobo caudado. Pp- Processo papilar do lobo caudado. Pc- Processo caudado do lobo caudado. H- Artéria hepática. Vb- Vesícula Biliar.

Em contrapartida, animais inseridos na mesma superordem do tatu, como a preguiça-comum (Bradypus variegatus) possuem também quatro lobos hepáticos sendo um lateral direito, medial direito, esquerdo e caudado. Onde, - lobo caudado não possui processos papilar e caudado, não há um lobo quadrado comum nas espécies domésticas e silvestres, e observou-se ausência de vesícula biliar (MACEDO, 2011). Outros animais silvestres como o preá 
SILVA, A.B.S. et al. Distribuição intraparenquimal da artéria hepática em tatu-peba (Euphractus sexcinctus) e tatu-verdadeiro (Dasypus novencinctus). PUBVET, Londrina, V. 8, N. 21, Ed. 270, Art. 1805, Novembro, 2014.

(Galea spixii), gambá (Didelphis albiventris), coypu (Myocastor coypus) e a capivara (Hydrochoerus hydrochaeris) possuem lobação hepática completa e vesícula biliar (NETO, 2000; OLIVEIRA et al., 2011; PEREZ, 2010; SOUZA, 2007;).

Já em animais domésticos, a distribuição dos lobos do fígado variam de acordo com as espécies, o cão, o gato e o suíno possuem lobação hepática completa, com exceção do suíno que não possui processo papilar do lobo caudado. No entanto, o ruminante possui apenas os lobos direito, esquerdo, quadrado e caudado. E o equino, apresenta lobo direito, quadrado, lateral e medial esquerdo, e processo caudado. Quanto a presença de vesícula biliar, o equino corresponde ao único, dentre os últimos citados, que é destituído de vesícula (GUETTY, 1986).

A artéria hepática dos tatus emite ramos que irão vascularizar o fígado, porções inicial e final do duodeno e pâncreas. Ao ingressar no hilo hepático, esta artéria apresenta-se distribuída em dois ramos principais: direito e esquerdo. No que concerne ao ramo direito na peça analisada, emitiu ramos destinados aos lobos quadrado, direito e caudado; e ainda emite ramos que irrigam a vesícula biliar. O ramo esquerdo dá origem a vasos destinados ao lobo esquerdo e quadrado (Figura. 2). Estes vasos que partem da artéria hepática se subdividem formando a microvascularização arterial hepática.

A vascularização arterial do fígado está estreitamente relacionada com a sua lobação, ou seja, animais com uma subdivisão lobular bem definida, como é o caso de carnívoros e súinos, tendem a apresentar maior quantidade de ramos que partem da artéria hepática para o parênquima. Carnívoros, apresentam três ramos principais, lateral e medial direito, e um ramo esquerdo. Em contrapartida, ruminantes, equinos e os tatus, apresentam apenas dois ramos que irão vascularizar o parênquima hepático, um ramo direito e um ramo esquerdo; fato que pode ser explicado pela locação menos pronunciada (GUETTY, 1986). Aspectos relacionados à lobação podem ter no tipo de dieta alimentar e no metabolismo próprio uma justificativa para o 
SILVA, A.B.S. et al. Distribuição intraparenquimal da artéria hepática em tatu-peba (Euphractus sexcinctus) e tatu-verdadeiro (Dasypus novencinctus). PUBVET, Londrina, V. 8, N. 21, Ed. 270, Art. 1805, Novembro, 2014.

desenvolvimento do órgão ser mais pronunciado em uns e menos definido em outros.

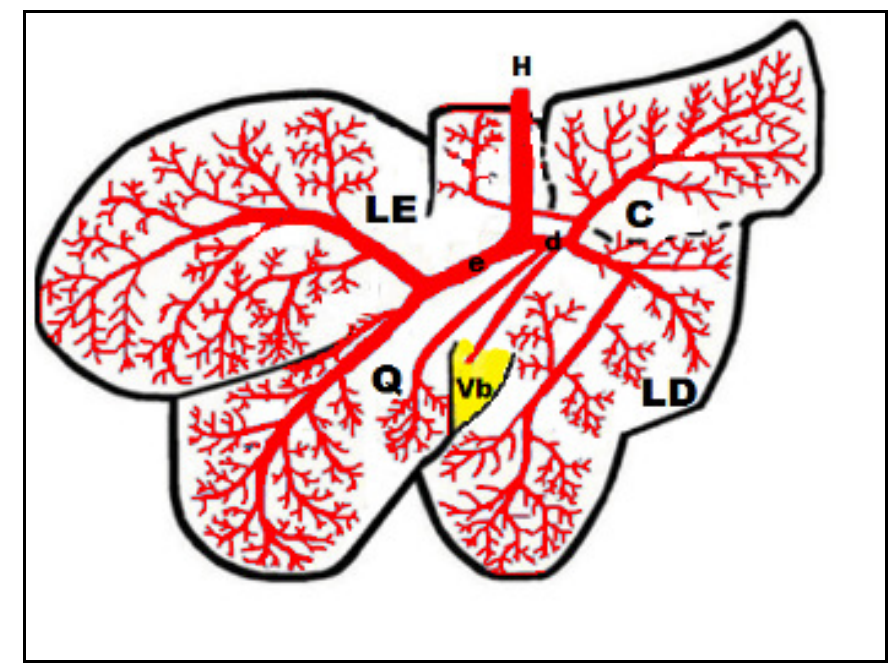

Figura 2. Representação da artéria hepática $(\mathrm{H})$ no fígado de tatus (Euphactus sexcintus e Dasypus novemcinctus), face visceral, mostrando os ramos: direito (d), fornecendo vasos para o lobo direito (LB), lobo caudado (C), vesícula biliar ( $\mathrm{Vb}$ ) e lobo quadrado (Q); e esquerdo (e) emitindo ramos para o lobo esquerdo (LE) e quadrado (Q).

\section{Conclusão}

A morfologia hepática dos tatus das espécies E.sexcinctus e D.novencinctus apresentou divergências quando comparada a outros animais silvestres e animais domésticos. O estudo da distribuição intraparenquimal da artéria hepática nestes animais, pode-se inferir a presença de zonas com vascularização arterial própria nos lobos hepáticos, caracterizando, portanto, a segmentação anátomo-cirúrgica arterial. Além disso, há estreita relação entre metabolismo e dieta alimentar com a morfologia hepática e a distribuição vascular para este órgão. 


\section{REFERÊNCIAS BIBLIOGRÁFICAS}

AZEVÊDO, L.M.; CARVALHO,M.A.M.; MENEZES, D.J.A.; MACHADO, G.V.; SOUSA, A.A.R.; XAVIER, F.G.; Distribuição intraparenquimal da artéria hepática em cutias (Dasyprocta sp, Rodentia). Braz. J. vet. Res. anim. Sci., São Paulo, v. 45, n. 1, p. 5-10, 2008.

BARBINO, M.T.; OLIVEIRA, C.M.; FONSECA, E.T.; FAVARON, P.O.; RODRIGUES, M.N.; MIGLINO, M.A.; Anatomia do fígado de fetos de Guinea Pig em final de gestação (Cavia porcellus [Linnaeus, 1758]). Biotemas, v.24; n.3; p.97-103, set., 2011.

BARBOZA, R.R.D.; A etnoecologia dos tatus-peba (euphractus sexcinctus (linnaeus, 1758) e tatu verdadeiro (dasypus novemcinctus linnaeus, 1758) na perspectiva dos povos do semiárido paraibano. Campina Grande, 2009. 178p. Dissertação (Mestrado de ciência e tecnologia e ambiental). Universidade Federal da Paraiba. Campina Grande - PB, 2009. 178p.

BORGES, E. M.; MACHADO, M. R. F.; OLIVEIRA, F. S.; SOUZA, W. M.; DUARTE, J. M. B. Aspectos morfológicos do fígado do cervo do pantanal (Blastocerus dichotomus). Brazilian Journal of Veterinary Research and Animal Science, São Paulo, v. 39, n. 2, p. 78-80, 2002.

DEPS, P.D.; ANTUNES, J.M.A.P.; FARIA, C. ; BÜHRER-SÉKUA, S.; CAMARGO, Z.P.; OPROMOLA, D.V.; TOMIMORI, J.; Research regarding anti-PGL-I antibodies by ELISA in wild armadillos from Brazil. Revista da Sociedade Brasileira de Medicina Tropical 41(Suplemento II). p.73-76, 2008.

FISCHER, P. F.; PETROCHI, D. L.; SANTOS, A. V.; SOUZA, J.; PINZON, P. W.; ARALDI, D. F.; Manejo nutricional da subordem Xenarthra. In: Seminário Interistintucional de Ensino Pesquisa e Extensão.16. Anais..Universidade no Desenvolvimento Regional. 2011. 4p.

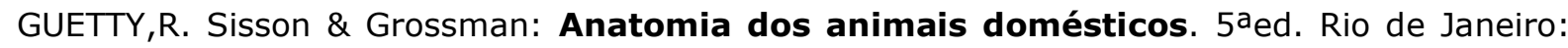
Guanaba Koogan, 2008. 2000p.

JUNQUEIRA, L. C.; CARNEIRO, J. Histologia básica. 11 ed. Rio de Janeiro: Guanabara Koogan, 2008. 524 p.

MACEDO, B.C.; BRANCO,E.;COSTA, A.M.; LIMA, A.R.; Lobação hepática da preguiça comum (Bradypus varegatus).. In: 38 Conbravet, 2011, Florianópolis. $38^{\circ}$ CONBRAVET Revista de Ciencias Agroveterinárias, 2011.

MENEZES, D.J.A.; CARVALHO, M.A.M.; CAVALCANTE FILHO, M.F.; SOUZA, W.M.; Configuração do sistema venoso portal na cutia (Dasyprocta aguti, RODENTIA). Braz. J. vet. Res. anim. Sci., São Paulo, v. 38, n. 6, p. 263-266, 2001.

NETO, R. A. F. Morfologia externa do fígado e principais ramificações da veia porta intrahepática no gambá (Didelphis albiventris). Salusvita, Bauru, v. 19, n. 2, p. 53-61, 2000.

OLIVEIRA, G.B.; RODRIGUES, M.N.; SILVA, R.S.B.; ALBUQUERQUE, J.F.G.; VALE, A.M.; MOURA, C.E.B.; OLIVERIA, M.F.; Lobação do fígado e distribuição intraparenquimal da veia porta no preá (Galea spixii Wagler, 1831). Biotemas, 24 (3): 105-110, set. 2011.

PEREIRA JUNIOR, H.R.J.; Evolução cromossômica na ordem Xenarthra. Botucatu, 2007. 181p. Tese (Doutorado em Ciências Biológicas). Universidade Estadual Paulista. Instituto de Biociências de Botucatu. Botucatu-SP, 2007. 181p. 
PÉREZ, W. \& LIMA, M. Anatomical description of the liver, hepatic ligaments and omenta in the coypu (Myocastor coypus). Int. J. Morphol., 25(1):61-64, 2007

SOUZA, W.N.; SOUZA, N.T.M.; CARVALHO, R.G.; CORREA, C.N.; Topografia da artéria hepática no fígado de capivara (Hydrochaerus hydrochaeris). Ciência Rural, v.37, n.1, janfev, 2007.

VIZCAINO, S.F.; LOUGHRY, W.J. The Biology of the Xenartha. University Press of Florida, Gainesville, F.L. 370p.

ZIMBRES, B.Q.C.; Efeito da fragmentação sobre a comunidade de tatus e tamanduás (Mammalia: Xenarthra) no Cerrado brasileiro: uma abordagem da ecologia de paisagens. Brasilia, 2010. 126p. Dissertação (Mestrado em Ecologia). Universidade de Brasília. Brasília - DF, 2010. 126p. 